\title{
Clinical Laboratory Regulation in the Philippines - Are we doing it right?
}

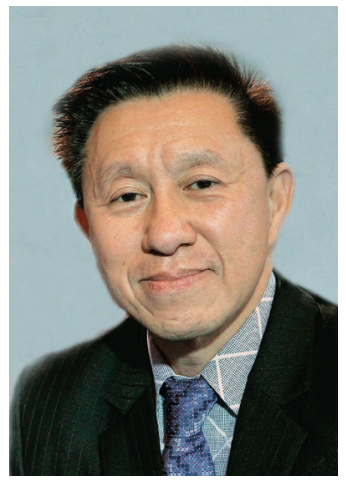

when I started my practice here.

I write this article from my perspective of 34 years of practice in the Philippines having arrived in 1987 from my training in the USA. In my six years of training first in Anatomic and Clinical Pathology then in Immunopathology fellowship, I have witnessed the workings of laboratory regulation and accreditation in that country and was in culture shock

Laboratory automation was well underway in the Western hemisphere during my training. Hematology and Chemistry at the forefront of this technological revolution. But other disciplines were starting to catch up. Locally, few tertiary labs were starting to automate. I was, of course, excited to begin revolutionizing the practice of Clinical Pathology.

I had not counted on the backward state of laboratory regulation locally. Under the Department of Health regulatory office, Bureau of Research and Laboratories (BRL), inspection visits to the tertiary labs that I was connected with (one private and one government hospital lab), the inspectors were engineers who came with a list of requirements that included asking for Benedict's reagent even though we were already using automated chemistry methods. This stopped only ten years ago, thankfully when the BRL was renamed Bureau of Health Facilities Services (BHFS), now the Health Facilities Services Regulatory Bureau (HFSRB).

The other issue is that there tends to be over regulation. Elsewhere, the emphasis is on accurate laboratory test results which is achieved through good quality assurance programs and performing satisfactorily on semi-annual proficiency testing. On-site inspections are geared towards these goals as well as the right physical set-up necessary to achieve these results. Our local regulatory agency tends to aim at including training programs which normally are the province of professional societies.

An example is HIV testing. When it was first proposed, you have to apply for a separate HIV testing license apart from the license to operate a clinical laboratory. To get the license, a medical technologist must have to attend and pass an HIV Proficiency training course which includes counselling patients. The course was being given by Research Institute of Tropical Medicine (RITM) on a limited basis, thus, only few medical technologists were licensed, severely limiting the number of labs that can perform HIV testing.

I had argued against these regulations. The HIV antibody (and subsequently the HIV antigen test) test was a routine serologic procedure that can be done on a manual or automated method either by EIA or ECLIA. To run these tests, one needs only be conversant with the requirements for running the samples like any other serologic examination. Secondly, the ruling ignores the role of the pathologist who is responsible for the test result ultimately. Third, the medical technologist is not the best person to be counselling patients. It is the attending physician who orders the test. Putting the burden of counselling on the med tech is a disservice to the patient and takes the med tech away from his/her main job, which is to run the tests.

We now have a situation where the regulatory agency prescribes training which is often unavailable but labs are expected to comply. The offshoot is that the few medical technologists who had the training became highly sought after to be able to put up HIV testing in clinical labs, leading to a black market where these techs offer their licenses for a fee.

The requirement for pre- and post-test counselling actually deterred patients from testing since it attaches a stigma to the disease which was what the regulation was supposed to remove. In other countries, one can simply walk into a lab and ask for the HIV test without any additional requirements. Some even offer anonymous testing.

All these factors: limited testing, counselling requirements and the attendant stigma attached may have led to the explosive increase in HIV cases we are seeing now in the Philippines.

Fast forward to today and not much has changed. With the SARS-CoV-2 pandemic, molecular laboratories for RT-PCR testing of the virus had to be set up quickly to enable adequate testing. Regulations governing the physical set up described a sample laboratory design with instructions on one way flow. It was not meant to be the template but when we submitted our design following the instructions, it was not allowed. No matter how we modified it, it all boiled down to just follow the sample design, no matter if the space configuration did not allow for it. That to and fro took all of one month, precious time wasted at the height of the pandemic. 
Training of pathologists was fortunately delegated to the Philippine Society of Pathologists Inc. (PSPI), rightly so since professional organizations should be conducting training and proficiency testing, as is being done in most countries.

Training of medical technologists was given to the RITM and the University of the Philippines (UP), both government institutions engaged in SARS-CoV-2 testing exclusively in the early part of 2020. Unfortunately, both were much too busy with the routine testing, and in fact were overwhelmed by the volume of tests requested that medical technologist training was done on a limited basis, further exacerbating the shortage of available testing facilities. There are a number of private institutions already doing molecular testing that could have been requested to take part in medical technologist training but were largely ignored. Even today, formal training by these two institutions is required of analysts though training courses are still very limited. Yet, it is a mandatory requirement for renewal of licenses to operate. This puts the laboratories in a bind. They can't get their staff trained due to the mandated institutions not offering the courses yet the regulatory agency rigidly requires it. Recently, the RITM has updated their training calendar and established a mechanism for recognizing additional training providers to catch up with the backlog of required trainings.

The result is that no additional shifts to accommodate more testing can be opened by the laboratories. They are limited to the staff that have been previously trained. In the early days of the pandemic, the HFSRB allowed labs with previously trained staff to train their new recruits. That is no longer allowed. It speaks of yet again an inability to trust the pathologist and med tech staff to competently train their own new techs, something that is being done in all sections of the clinical laboratory. The RT-PCR technique has been elevated to an esoteric technique that requires formal training, like what was done with HIV testing earlier.

Now we are witnessing an effort by $\mathrm{DOH}$ to formulate new regulations for other molecular testing in all its forms: infectious disease, cancer and genetic. Yet we have been practicing molecular pathology since the early 1990s. It is no longer exotic and is actually

Dr. Raymundo Lo is a respected pathologist of Philippine Children's Medical Center, an immunopathologist of St. Luke's Medical Center, Quezon City, a member of the Philippine Society of Pathologists, Inc., a columnist of the Manila Bulletin, an editorial board member of the Philippine Journal of Pathology, and a member of the COVID-19 Laboratory Experts Panel (CLEP) of the Department of Health. becoming part and parcel of clinical laboratory testing, much like ELISA and ECLIA were once deemed so advanced as to merit special training for HIV.

Many molecular tests are now very easy to perform. Cartridge-based molecular testing can even be done at point of care. Even Anatomic pathology practitioners do in-situ hybridization routinely for breast and other cancers. An offshoot of cancer molecular testing is that once identified, the protein that the gene codes for can also be targeted with immunohistochemistry.

Molecular testing is the future of clinical laboratory testing. If misguided regulations are put in effect, that may adversely affect progress of laboratory testing in the Philippines. We hope the DOH learns the lessons of the past so as not to repeat it in the future.

The regulatory agencies being burdened with many tasks and being short of technical staff should delegate tasks that can be done by others. It should coordinate with RITM and other training providers to see if the assigned training tasks can be done properly and in due time. It should also tap private entities that are as capable as these agencies in trainings

There is hope in the establishment of the Office for Health Laboratories ( $\mathrm{OHL}$ ) which is headed by a pathologist. Since regulation will still be with the HFSRB, there should be regular meetings with the $\mathrm{OHL}$, the PSPI and Philippine Association of Medical Technologists (PAMET) for updated implementation of regulatory policies based on current clinical laboratory progress in technology and knowledge.

In addition, the $\mathrm{DOH}$ should further strengthen its collaborative work with PSPI and PAMET in ensuring a smooth flow of licensing and compliance. The PSP and PAMET should be tapped for its members to assist the $\mathrm{DOH}$ inspection teams which will improve the process by better communication with the laboratory staff during inspection.

Currently, a Technical Working Group on Molecular Laboratory testing is working on the future provisions of future regulations. We wish it success for the seamless adaptation of molecular testing by local laboratories.

\section{Raymundo W. Lo, MD, FPSP}

Anatomic and Clinical Pathologist, Philippine Children's Medical Center, Quezon City, Philippines

https://doi.org/10.21141/PJP.2021.19 